\title{
LONGITUDINAL AND TRANSVERSE DEVELOPMENT OF ELECTROMAGNETIC SHOWERS USING SILICON DETECTORS
}

\author{
SICAPO Collaboration \\ F. Lemeilleur \\ CERN, Geneva, Switzerland \\ F. Lamarche and C. Leroy \\ McGill University, Montreal, Canada \\ R. Paludetto, S. Pensotti ${ }^{*}$, P.G. Rancoita and L. Vismara \\ INFN, Milan, Italy \\ A. Seidman \\ University of Tel Aviv, Israel
}

\begin{abstract}
Extensive experimental studies of longitudinal and transverse development of electromagnetic showers initiated by electrons of $2-49 \mathrm{GeV}$, using sampling calorimeters with silicon detectors as active layers, are reported.
\end{abstract}

Presented at the

International Conference on Advanced Technology and Particle Physics

Como, Italy, 13-16 June 1988

*) Also at University of Milan, Italy. 


\section{INTRODUCTION}

In the past few years the SICAPO (SIlicon CAlorimeter and POlarimeter) Collaboration (Florence, Hamburg, McGill, Messina, Milan, Oregon, Tel Aviv, Tennessee, Trieste) has contributed to the development of large-area silicon detectors as active layers in sampling calorimeters. Measurements were performed extensively in order to study the longitudinal and the lateral development of electromagnetic (e.m.) showers. Experimental results are presented on the mean energy detected and the energy resolution as a function of the incoming electron energy, and the longitudinal energy detected as a function of the calorimeter depth and the silicon detector depletion widths. The measurements of the transverse e.m. shower development using a silicon strip detector are reported for several shower depths. Tungsten and uranium are used as high-Z absorbing materials. The attenuation of the sensed energy by introducing hydrogeneous low- $Z$ absorber in various configurations is presented. The size of the detectors and the beam definition have been chosen so as to allow a large shower containment. Measurements were performed from 2 to $49 \mathrm{GeV}$ using electrons from the CERN Proton Synchrotron (PS) and Super Proton Synchrotron (SPS) beams, which have a momentum resolution from $3 \%$ at $49 \mathrm{GeV}$ to $1 \%$ at lower energies.

\section{LONGITUDINAL DEVELOPMENT}

The experimental set-up is shown in fig. 1. Twelve silicon detectors $5 \times 5 \mathrm{~cm}^{2}$ in area are used interleaved with 2 radiation lengths $\left(\mathrm{X}_{0}\right)$ of tungsten or uranium. The electron-beam trigger is defined by the coincidence of a Cherenkov, two scintillators SC1 $\left(10 \times 10 \mathrm{~cm}^{2}\right)$ and SC2 $\left(0.5 \times 0.5 \mathrm{~cm}^{2}\right)$, and one small silicon detector $\left(0.5 \times 0.5 \mathrm{~cm}^{2}\right)$ placed just in front of the calorimeter and centred on its longitudinal axis. In this way an electron impinging at $\pm 0.25 \mathrm{~cm}$ in the centre of the calorimeter has its shower fully contained transversally $(>95 \%)$ in 5 Molière radii (1 Molière radius is equal to $0.9 \mathrm{~cm}$ for $\mathrm{W}$ and $0.95 \mathrm{~cm}$ for $\mathrm{U}$ ). The mean energy $\epsilon$ in $\mathrm{MeV}$ detected by the 12 detector planes depleted at $200 \mu \mathrm{m}$ for $\mathrm{W}$ absorber is shown in fig. 2 as a function of the incoming electron energy $\mathrm{E}$ in $\mathrm{GeV}$. The line is the least-squares fit to the data,

$$
\epsilon=(5.558 \pm 0.004) \mathrm{E}+(-1.3 \pm 1.5)[\mathrm{MeV} / \mathrm{GeV}]
$$

and indicates a very good linearity of the energy response. The energy resolution of a calorimeter is dominated by sampling fluctuations and is given by

$$
\sigma / \mathrm{E}=\mathrm{k}(\tau / \mathrm{E})^{1 / 2},
$$

where $\tau$ is the sampling frequency in radiation lengths of the passive material, and $\mathrm{E}$ the incoming energy in GeV. Figure 3 shows the values of $k$ obtained as a function of the beam energy; the dashed line indicates the weighted mean of

$$
\mathrm{k}=17.6 \pm 0.3 \%
$$

The longitudinal shower development is measured by recording the deposited energy $\epsilon$ in each active plane. This is shown for $\mathrm{W}$ in fig. 4 as a function of the calorimeter depth $\mathrm{t}$ in radiation lengths and for $4,9,16,25$, and $49 \mathrm{GeV}$ incident energies. The shape of the development follows, up to $12 \mathrm{X}_{0}$, the empirical equation

$$
\epsilon=\epsilon_{0} \mathrm{t}^{\mathrm{a}} \exp (-\mathrm{bt})
$$


where a has a logarithmic dependence on the energy, $b$ is a constant, and $\epsilon_{0}$ is a normalizing constant. A better parametrization up to $24 \mathrm{X}_{0}$ has been found [1] and the results are indicated by the curves of fig. 4 . While the above results are obtained for detectors at a fixed depletion depth of 200 $\mu \mathrm{m}$, the following concerns the calorimeter-collected energy for various depletion depths $X_{d}$ obtained by adjusting the detector bias voltages $\mathrm{V}$ :

$$
\mathrm{X}_{\mathrm{d}}=0.529 \times 10^{-4}\left[\varrho\left(\mathrm{V}+\mathrm{V}_{\mathrm{b}}\right)\right]^{1 / 2}(\mathrm{~cm})
$$

where $Q$ is the silicon detector resistivity and $V_{b}$ the built-in voltage. Figure 5 shows the total energy sensed by the calorimeter as a function of $X_{d}$ from 40 to $200 \mu \mathrm{m}$ for a $4 \mathrm{GeV}$ electron beam. The response is linear over the whole range measured. However, for a zero bias voltage the sensed energy is still positive. It is interpreted as charge diffusion from the field-free region into the intrinsic depletion region due to the built-in voltage $\mathrm{V}_{\mathrm{b}}$ close to the detector junction. This effect is estimated to give an effective extra charge collection over $23 \pm 2 \mu \mathrm{m}$ at zero depletion depth. The energy sensed by individual planes as a function of the depletion depth is shown in fig. 6 for $4 \mathrm{GeV}$ incident electrons. The energy response from detectors located at 2 to 16 radiation lengths is linear with the depletion layer width, while it is highly non-linear for those located at 18 to 24 radiation lengths. However, the overall linear calorimeter response is not altered by this effect owing to the small fraction of energy deposited at large depth in the calorimeter. Figure 7 shows the energy response of the calorimeter as a function of the incoming electron energy for 70 and $200 \mu$ m detector depletions and for $\mathrm{W}$ and $\mathrm{U}$ as absorbing material. The dependence of the calorimeter response on the incident energy is linear for the two depletion depths and for the two absorbers. The mean detected energy with $\mathrm{U}$ is about $11 \%$ higher than for $\mathrm{W}$ and the energy resolution is degraded by $10-15 \%$ for a depletion of $70 \mu \mathrm{m}$ compared to that already mentioned for a depletion of $200 \mu \mathrm{m}$. The influence of introducing hydrogeneous absorbers on the visible energy of the calorimeter is discussed elsewhere [2].

\section{TRANSVERSE DEVELOPMENT}

Silicon-strip detectors were used for the transverse e.m. shower development studies. Figure 8 shows the experimental set-up. The coincidence of signals coming from a Cherenkov, a large $10 \times 10$ $\mathrm{cm}^{2}$ scintillator $\mathrm{SC} 1$, a small $0.5 \times 0.5 \mathrm{~cm}^{2}$ scintillator $\mathrm{SC} 2$, and a strip detector SD1 with vertical strips selects electrons impinging in the longitudinal axis of the calorimeter with an accuracy of $\pm 0.25 \mathrm{~cm}$ in the vertical direction and $\pm 0.04 \mathrm{~cm}$ in the horizontal one. The calorimeter itself consists of a fixed strip detector SD2 with a $1 \mathrm{~mm}$ pitch as active plane and of absorber plates which can be placed on each side of the detector. Figure 9 shows the various configurations which have been used for the arrangements of the detector relative to the absorber.

in configuration $A$, high- $\mathrm{Z}$ absorber plates are placed exclusively in front of the detector with an air gap of $\mathrm{d}_{1}=10 \mathrm{~mm}$;

in configuration $B$, high- $\mathrm{Z}$ absorber plates are placed on both sides of the detector with air gaps of $\mathrm{d}_{2}=1.6 \mathrm{~mm}$ and $\mathrm{d}_{3}=1.4 \mathrm{~mm}$ in front and at the rear of the detector respectively;

in configuration $C$, each $2 \mathrm{X}_{0}$ of high- $\mathrm{Z}$ absorber is interleaved with one plane of low- $\mathrm{Z}$ material (G10), one plane of G10 being placed directly after the detector (same air gaps as for configuration $\mathrm{B}$ );

in configuration $D$, which is rather similar to configuration $C$, one radiation length of high- $Z$ material is placed directly after the detector. 
Figure 10 shows the energy sensed by each strip of the detector as a function of the number of $\mathrm{X}_{0}$ of absorber in front of it and obtained with configuration $\mathrm{A}$ at 2,4 , and $6 \mathrm{GeV}$ incident electron energies. The results are obtained using the 24 central strips of a $2.8 \times 2.8 \mathrm{~cm}^{2}$ detector with a $1 \mathrm{~mm}$ pitch and placing in front of it, successively 2 to 16 (by steps of 2) $X_{0}$ of high-Z absorber [3]. The comparison of the results for W (Fig. 10a) and U (Fig. 10b) shows that the deposited energies in the silicon strips are on an average $16 \%$ higher for $U$ than for $W$ for the three beam energies. The full width at half maximum of the summed distributions along $\mathrm{X}_{0}$ is $6 \mathrm{~mm}$ for both $\mathrm{W}$ and $\mathrm{U}$ at $2 \mathrm{GeV}$, $5 \mathrm{~mm}$ for $\mathrm{W}$ and $6 \mathrm{~mm}$ for $\mathrm{U}$ at $4 \mathrm{GeV}$, and $5 \mathrm{~mm}$ for both $\mathrm{W}$ and $\mathrm{U}$ at $6 \mathrm{GeV}$. The distributions show that the showers develop a narrow central part containing most of the incident energy corresponding to energy deposition by fast electrons, and a broad lateral part due to photons and slow electrons scattered away from the shower axis. A first attempt at fitting these distributions is described in ref. [4]. The preliminary experimental results presented in figs. 11, 12, and 13 are obtained for a $6 \mathrm{GeV}$ electron beam energy using respectively the configurations $\mathrm{B}, \mathrm{C}$, and $\mathrm{D}$ with a larger strip detector $\left(4.8 \times 4.8 \mathrm{~cm}^{2}\right)$ than before, which has 48 strips with $1 \mathrm{~mm}$ pitch. Figures $11 \mathrm{a}$, $12 \mathrm{a}$ and 13a show, in tridimensional plots, the energies sensed (vertical axis in arbitrary units) by the 48 strips ( $\mathrm{S}$ axis in $\mathrm{mm}$ ) for 2 to $12 \mathrm{X}_{0}$ of $\mathrm{W}$ absorber in steps of $2 \mathrm{X}_{0}$. The distributions are normalized according to the indicated vertical scale. Figures $11 \mathrm{~b}, 12 \mathrm{~b}$, and $13 \mathrm{~b}$ present the same results in a way which better emphasizes the evolution of the lateral distributions from 2 to $12 \mathrm{X}_{0}$ (top to bottom). The maximum of each distribution is normalized according to the indicated vertical scale. The purpose of introducing a hydrogeneous absorber (G10) after the detector is to attenuate the low-energy back-scattered shower component [2]. This attenuation is clearly shown by fig. 12 ( $5 \mathrm{~mm}$ of G10 at the rear of the detector), where the energy sensed is about $29 \%$ lower than the energy sensed without G10 (fig. 11). The attenuation of the energy sensed is about $10 \%$ smaller (fig. 13) when the same amount of G10 is separated by one $\mathrm{X}_{0}$ of $\mathrm{W}$ from the rear of the detector.

\section{CONCLUSION}

Impressive progress has been made in the use of silicon detectors as the active medium in calorimeters. The response of a sampling calorimeter built with such detectors is linear with the incident electron energy, and an energy resolution of $17.6 \pm 0.3 \%(\tau / \mathrm{E})^{1 / 2}$ has been achieved. The silicon detectors can be used not fully depleted, leading to the use of low-resistivity material at rather low bias voltages.

\section{Acknowledgements}

We thank E. Borchi, M. Bruzzi, C. Furetta, A. Penzo, C. Simeone and L. Venturelli for their help in data taking and operations, and for constructive discussions. 


\section{REFRENCES}

[1] G. Barbiellini et al., Nucl. Instrum. Methods A235 (1985) 216;

G. Barbiellini et al., Nucl. Instrum. Methods A235 (1985) 55;

G. Barbiellini et al., Nucl. Instrum. Methods A236 (1985) 316;

G. Barbiellini et al., Nucl. Instrum. Methods A240 (1985) 289.

[2] SICAPO Collaboration (presented by C. Leroy), Silicon sampling hadronic calorimetry: a tool for experiments at next generation colliders, these Proceedings.

[3] F. Lemeilleur, P.G. Rancoita and A. Seidman, IEEE Trans. Nucl. Sci. NS34 (1987) 538.

[4] G. Ferri et al., Nucl. Instrum. Methods A273 (1988) 123. 


\section{Figure Captions}

Fig. 1 Experimental set-up for longitudinal e.m. shower studies.

Fig. 2 Mean energy detected by the calorimeter as a function of the incoming electron energy with $\mathrm{W}$ absorber for $200 \mu \mathrm{m}$ silicon detector depletion.

Fig. 3 The $k$ values in $\%$; 0 : data for the off-line sum; $\bullet$ : data for the hardware sum.

Fig. 4 Longitudinal shower development as a function of the depth $t$ in number of radiation lengths for $4,9,16,25$, and $49 \mathrm{GeV}$ with $\mathrm{W}$ absorber. The lines are the fitted curves.

Fig. 5 The energy response of the calorimeter with $\mathrm{W}$ absorber as a function of the silicon detector depletion depths $X_{d}$ at $4 \mathrm{GeV}$. The line is a least-squares fit of the data.

Fig. 6 The longitudinal shower development for silicon detector depletion depths of 40, 70, 100, 125,150 , and $200 \mu \mathrm{m}$ with $\mathrm{W}$ absorber at $4 \mathrm{GeV}$. The curves are the linearized least-squares fits of the data.

Fig. 7 Mean energy detected as a function of the incoming electron energy at a silicon detector depletion of $200 \mu \mathrm{m}$ for $\mathrm{W}(\bullet)$ and $U(\bullet)$, and $70 \mu \mathrm{m}$ for $\mathrm{W}(\circ)$ and $U(\square)$. The full lines are the least-squares fits of the data.

Fig. 8 Experimental set-up for transverse e.m. shower studies; c: Cherenkov; SC1: $10 \times 10 \mathrm{~cm}^{2}$ scintillator; SC2: $5 \times 5 \mathrm{~mm}^{2}$ scintillator; SD1: $200 \mu \mathrm{m}$ pitch strip detector for horizontal beam definition; SD2: $1 \mathrm{~mm}$ pitch strip detector for transverse shower measurement; Abs: Absorber.

Fig. 9 Arrangements for the absorber and silicon strip detector SD2 for transverse e.m. shower studies. Configuration $\mathrm{A}$ : high- $\mathrm{Z}$ absorber only in front of SD2; configuration $\mathrm{B}$ : high- $\mathrm{Z}$ absorber in front and at the rear of SD2; configuration $C$ : similar to configuration $B$ with introduction of $\mathrm{G} 10$ each $2 \mathrm{X}_{0}$ and a plate of $\mathrm{G} 10$ at the rear of $\mathrm{SD} 2$; configuration $\mathrm{D}$ : similar to configuration $\mathrm{C}$ with one $\mathrm{X}_{0}$ of high- $\mathrm{Z}$ absorber at the rear of SD2.

Fig. 10 Lateral shower distributions obtained with configuration $A$ for 2 to 16 radiation lengths $X_{0}$ (by steps of $2 \mathrm{X}_{0}$ ), $\mathrm{S}$ being the $1 \mathrm{~mm}$ pitch strip number; a) for $\mathrm{W}$ at 2,4 , and $6 \mathrm{GeV}$; b) for $\mathrm{U}$ at 2,4 , and $6 \mathrm{GeV}$.

Fig. 11 Lateral shower distributions obtained with configuration $B$ for 2 to $12 X_{0}$ (by steps of $2 X_{0}$ ) $\mathrm{S}$ being the $1 \mathrm{~mm}$ pitch strip number. a) tridimensional distributions; $\mathrm{b}$ ) distributions from $2 \mathrm{X}_{0}$ (top) to $12 \mathrm{X}_{0}$ (bottom).

Fig. 12 Lateral shower distributions obtained with configuration C. Same comments as for the previous figure.

Fig. 13 Lateral shower distributions obtained with configuration D. Same comments as for previous figure. 


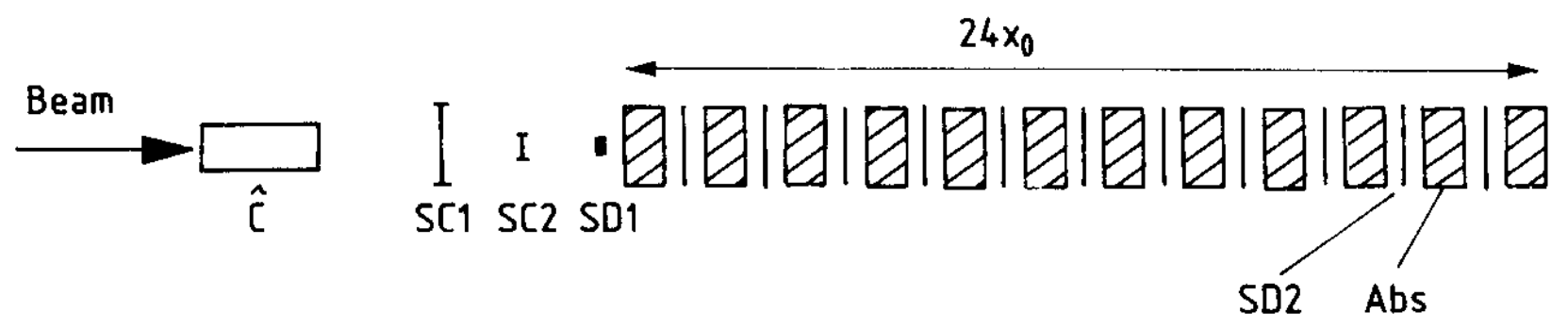

Fig. 1

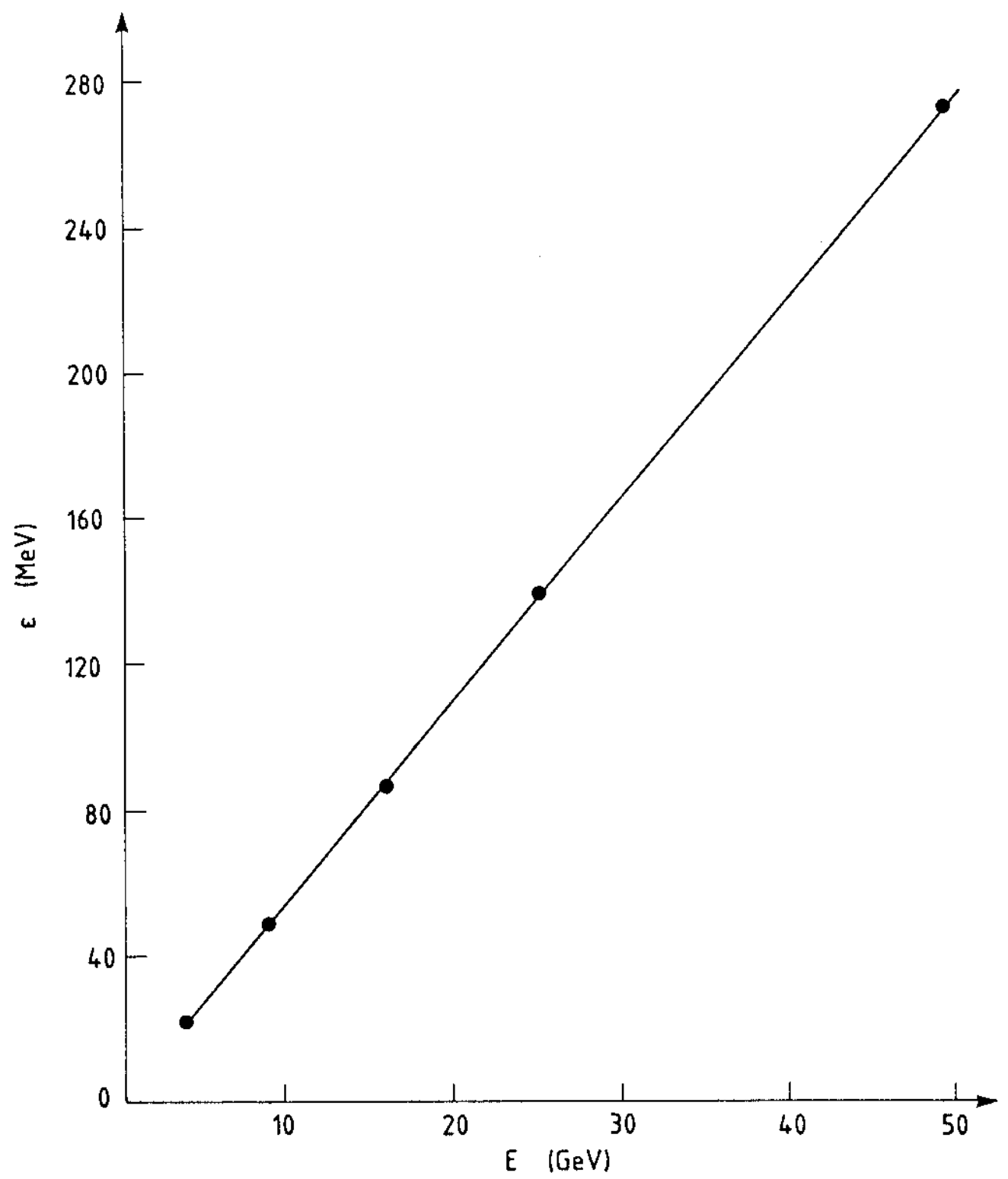

Fig. 2 


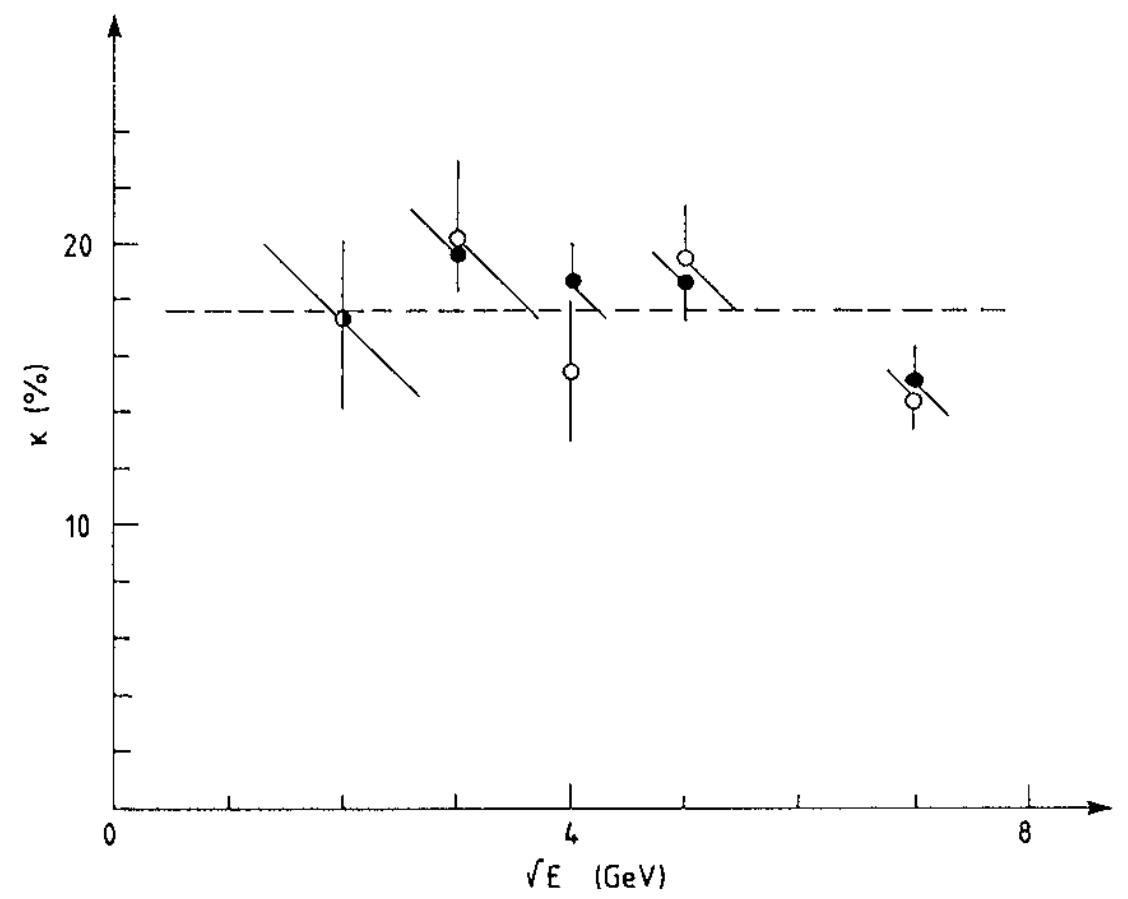

Fig. 3

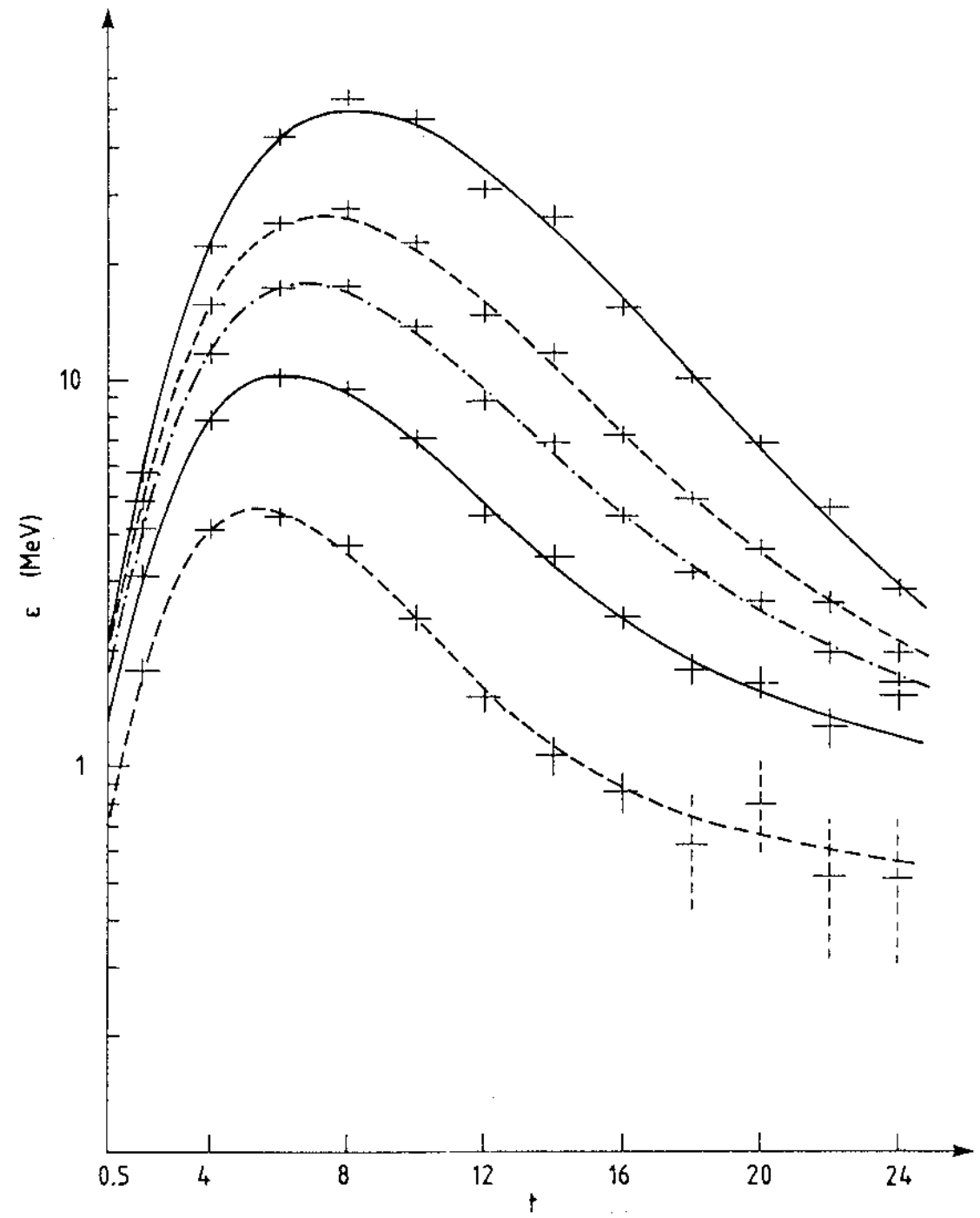

Fig. 4 


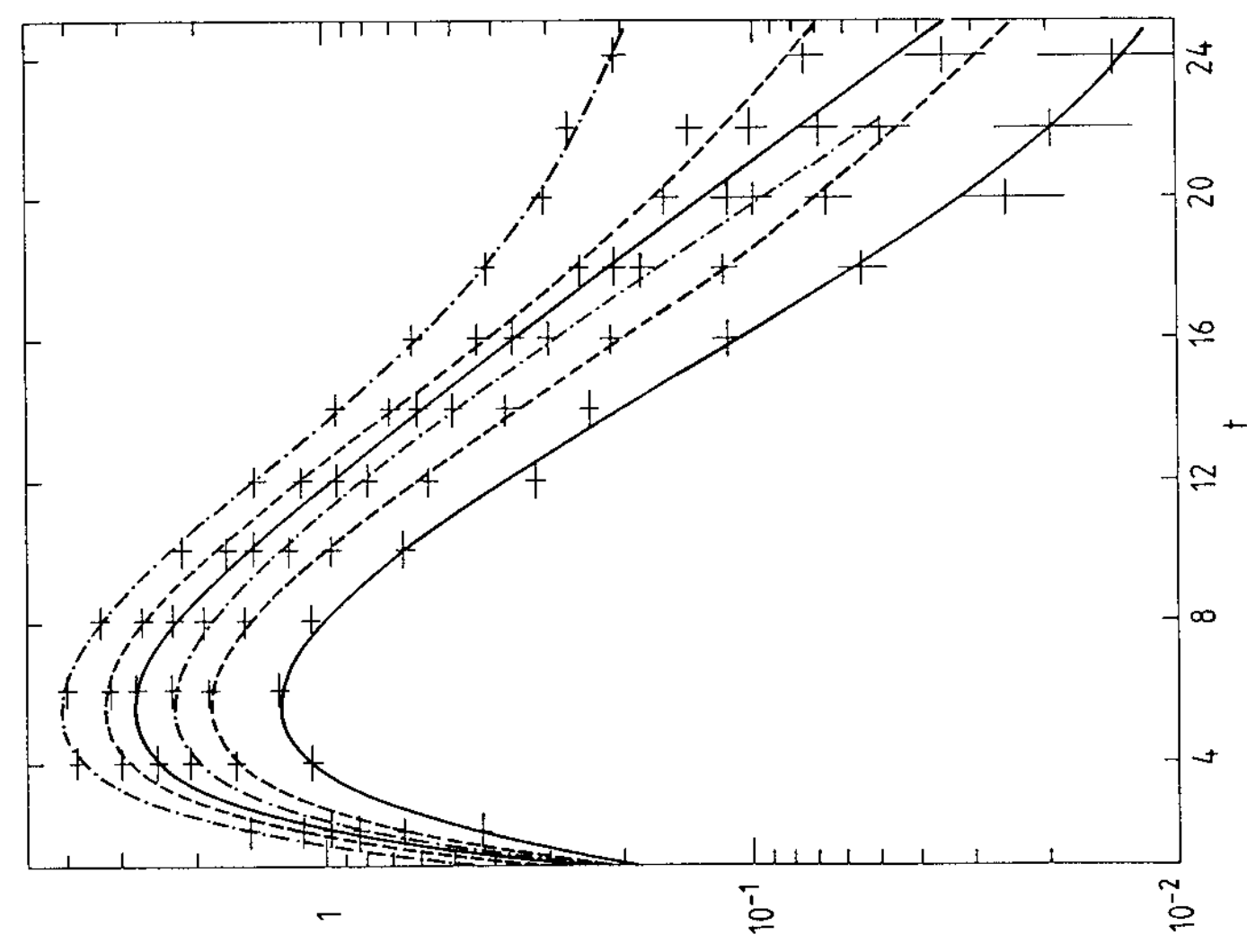

$\left(\Lambda^{2} W\right)^{3}$

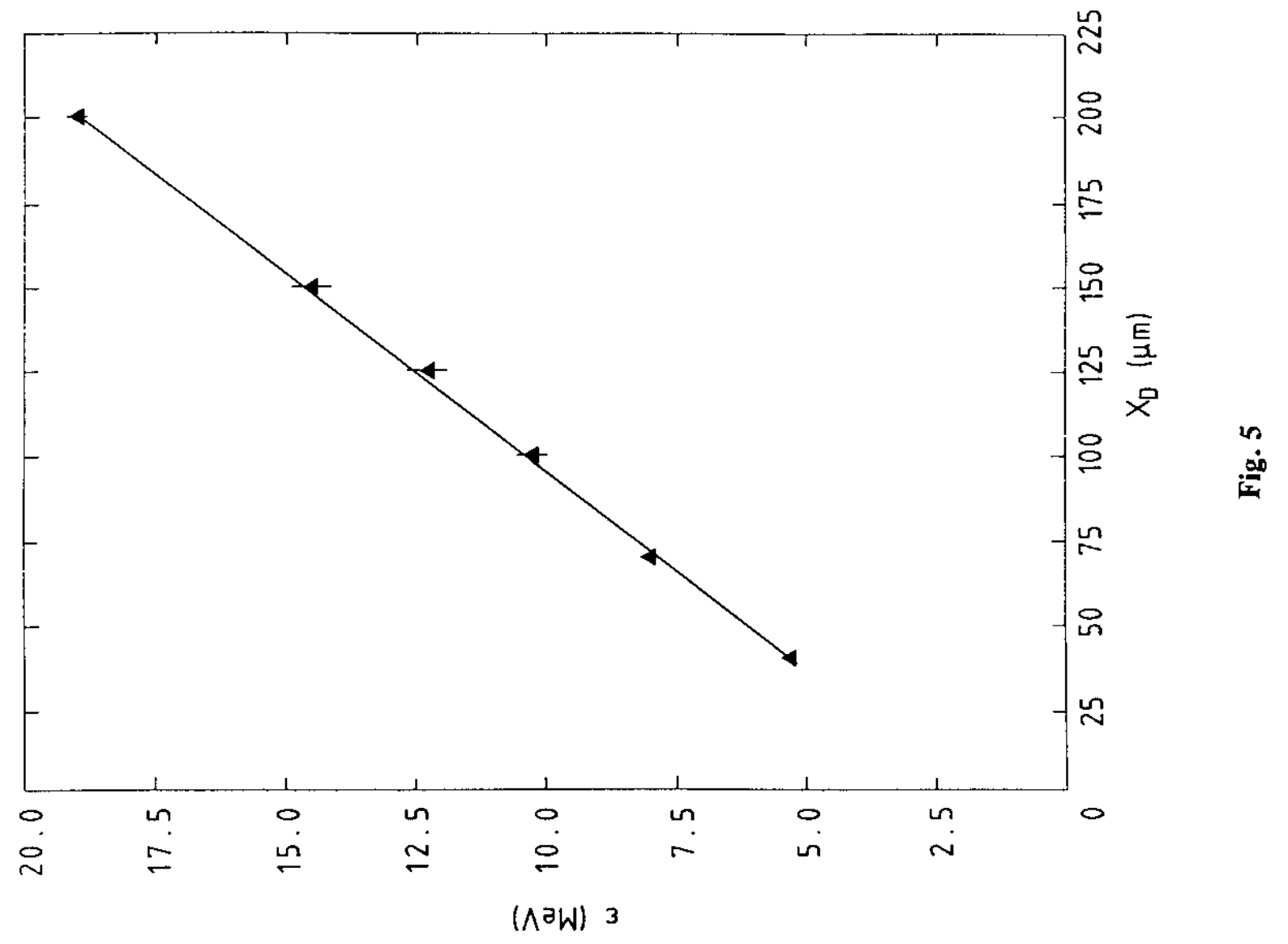




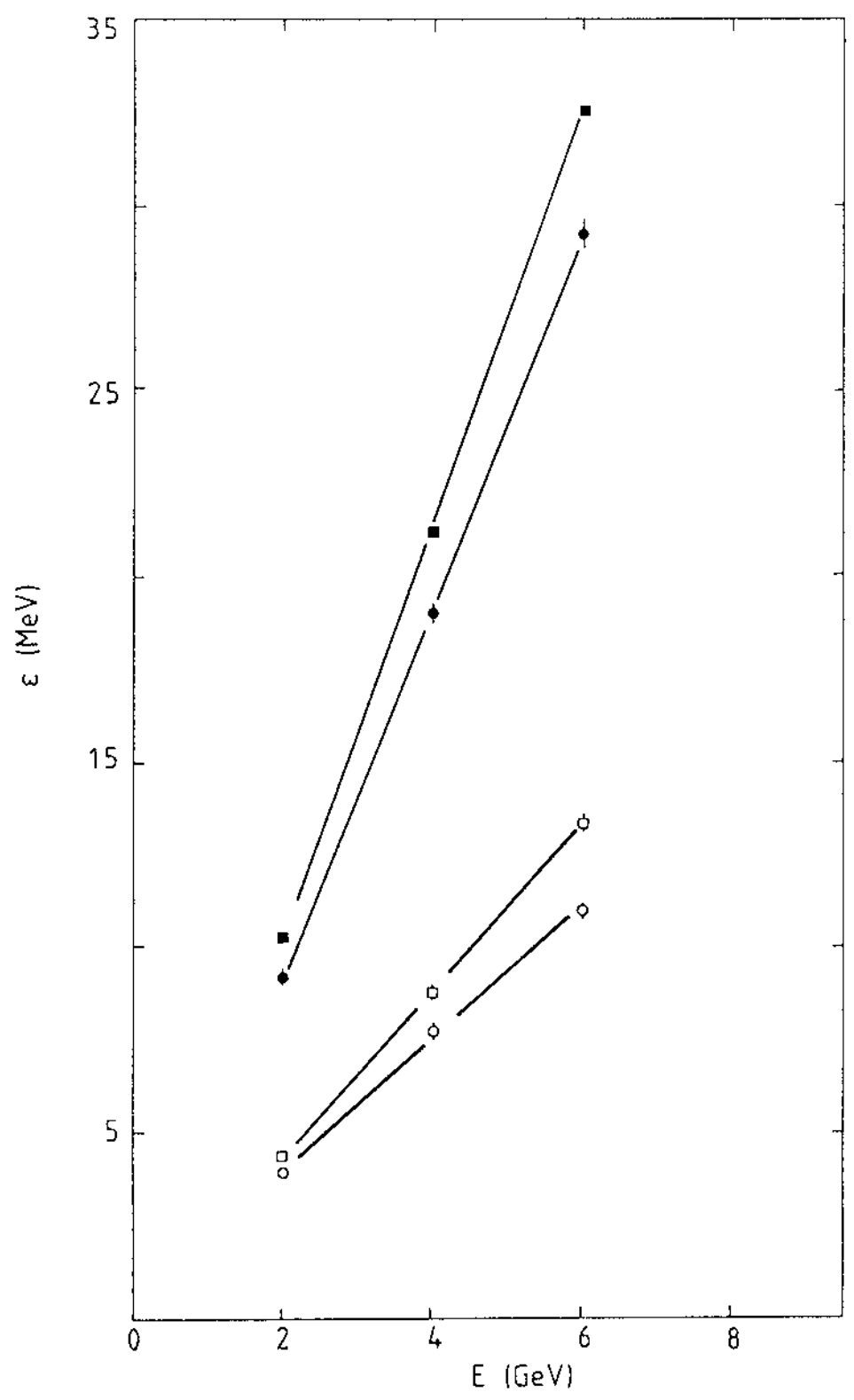

Fig. 7

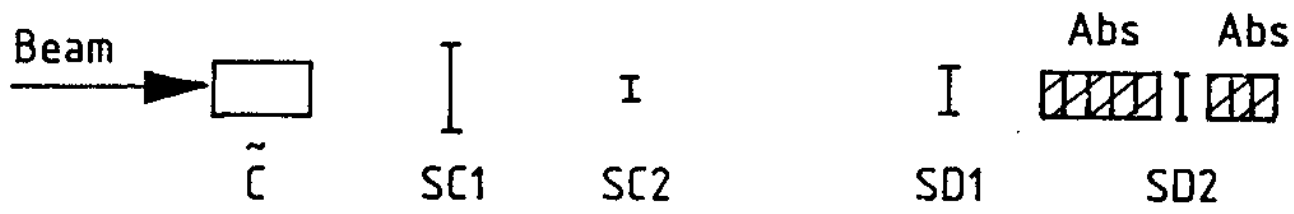

Fig. 8 

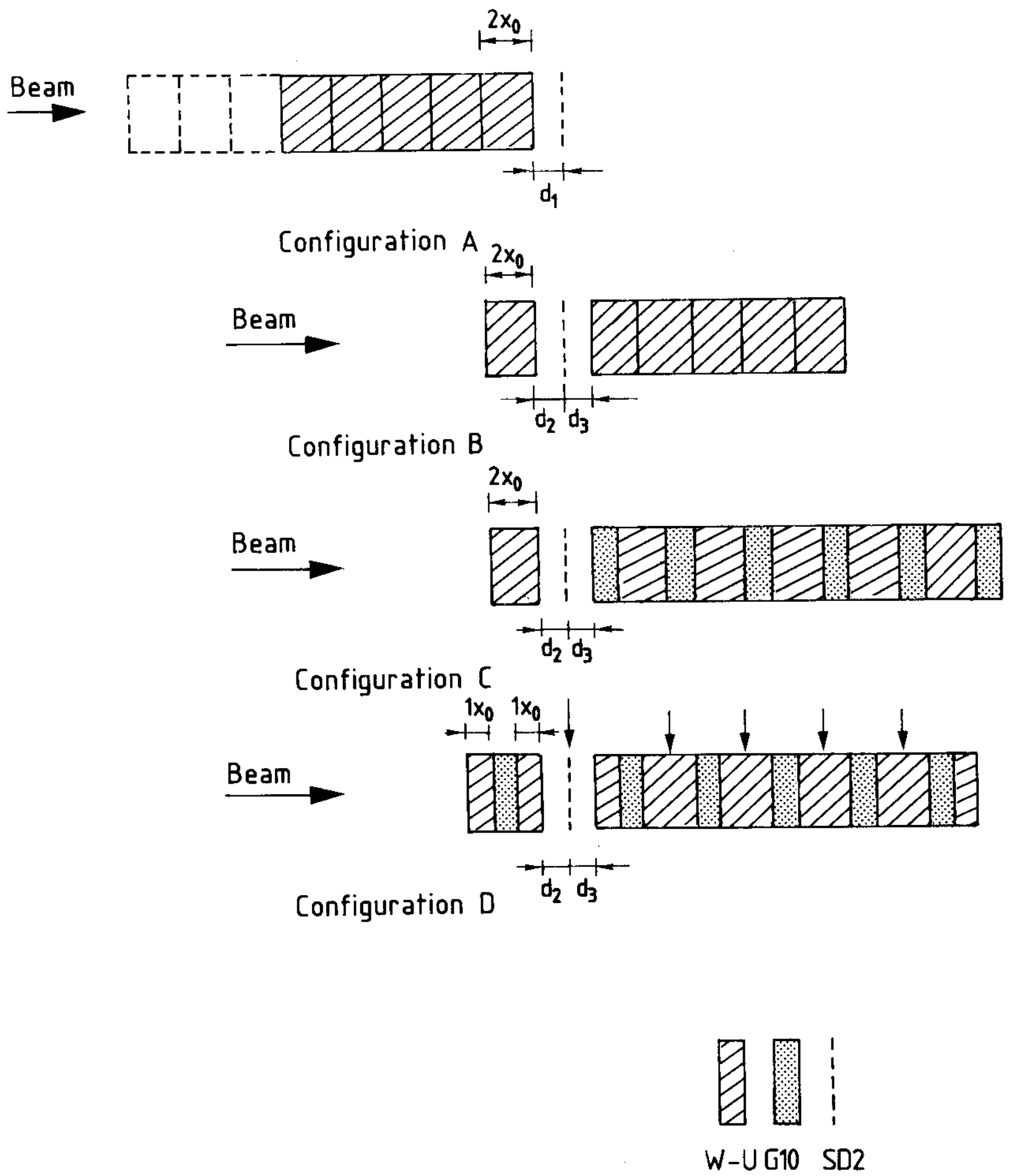

Fig. 9 

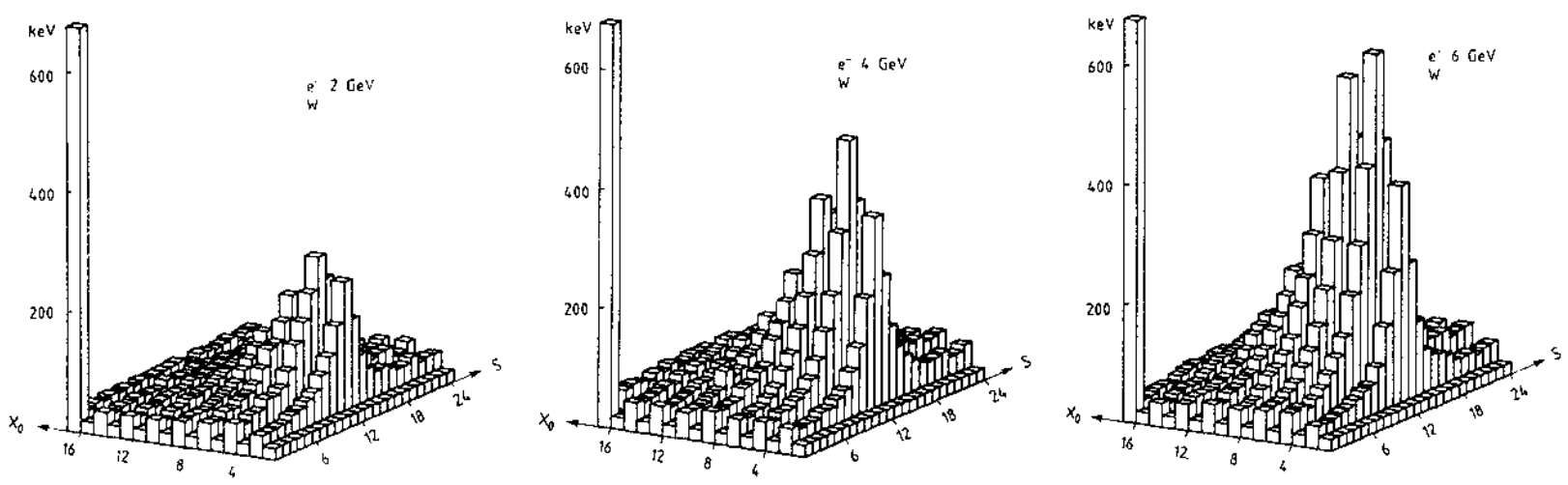

a)
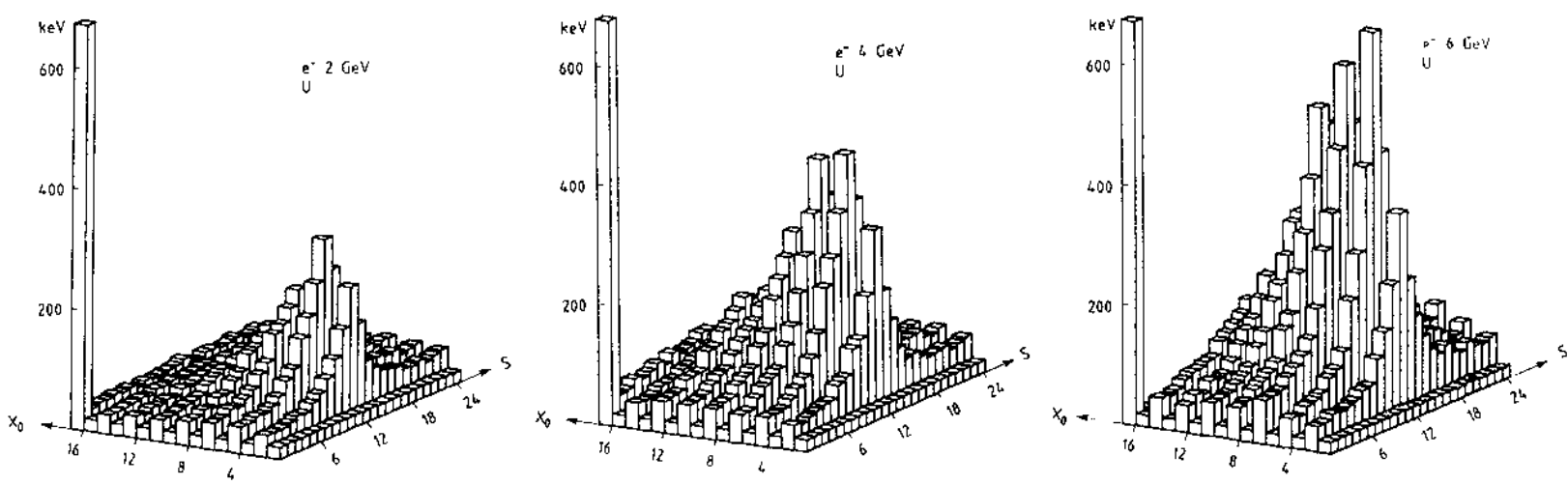

b)

Fig. 10 
Fig. 11

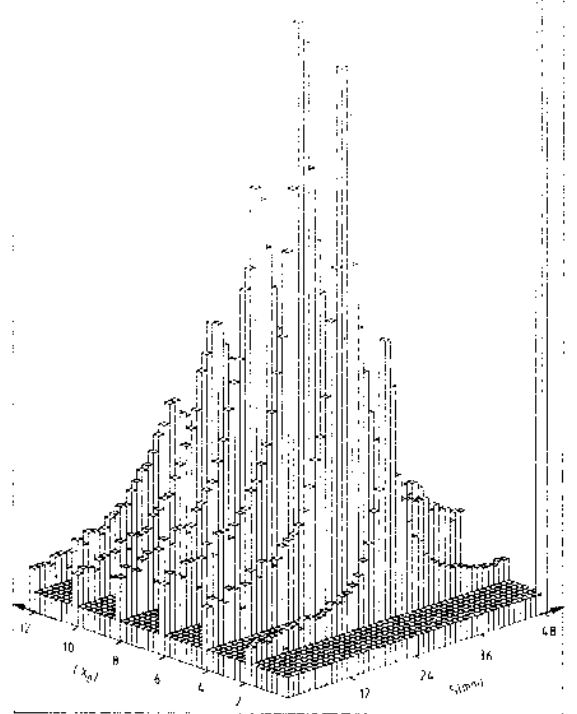

a) b)

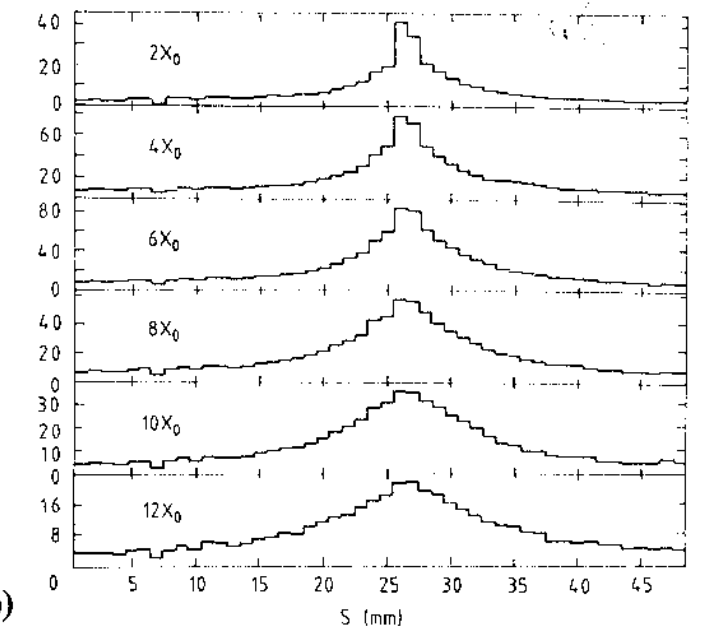

Fig. 12

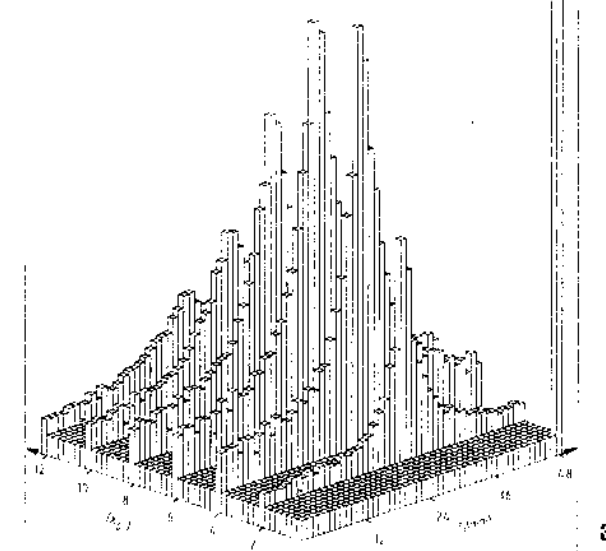

a) b)

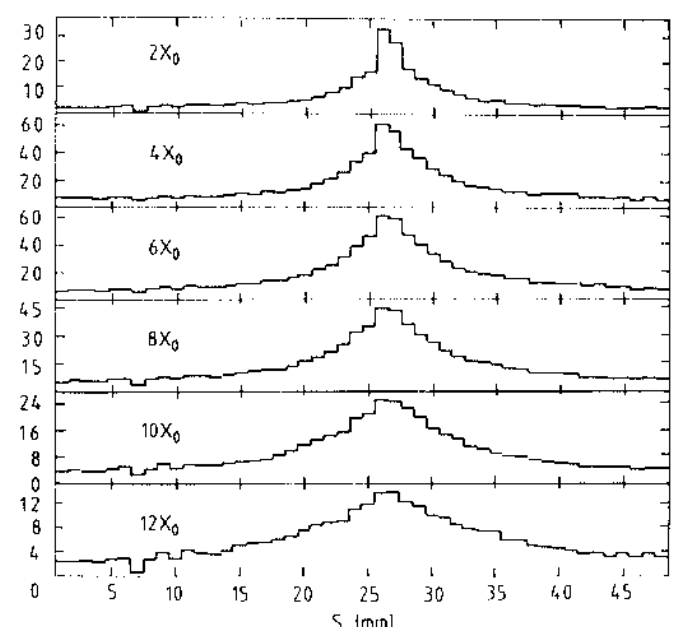

Fig. 13

a)

b)

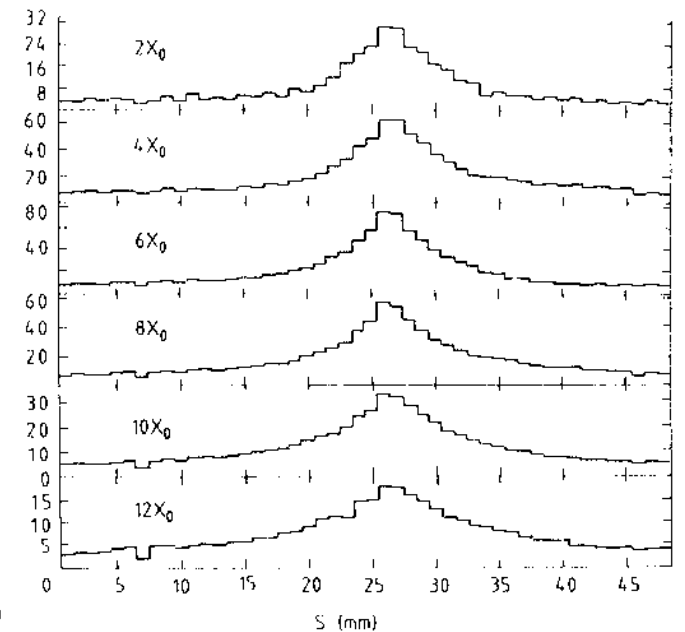

